\title{
Estimation de durées de vie de systèmes mécaniques complexes par essais accélérés
}

\author{
Pascal Lantiéri $^{1, a}{ }^{\text {, Fabrice Guérin }}{ }^{2}$ et Ridha Hambli ${ }^{3}$ \\ 1 Arts \& Métiers ParisTech, LPMI, 2 boulevard du Ronceray, 49100 Angers, France \\ 2 Université d'Angers, LASQUO, 62 avenue ND du Lac, 49000 Angers, France \\ 3 Polytech'Orléans, LMSP, 8 rue Léonard de Vinci, 45072 Orléans Cedex 2, France
}

Reçu le 28 août 2008, accepté le 4 novembre 2008

\begin{abstract}
Résumé - Nous proposons dans cet article une méthode d'essai accéléré applicable pour des systèmes mécaniques complexes dont la durée de vie ne peut être exprimée par une relation analytique du niveau de sollicitation. Dans de tels cas, une approche numérique peut rester envisageable. Il s'agit alors d'ajuster le modèle numérique à partir de tests sévérisés au cours desquels les défaillances apparaissent plus rapidement qu'en conditions nominales. Il est ainsi possible de prévoir à partir d'essais très courts des défaillances susceptibles d'apparaître après une longue période d'utilisation. Cette méthode est mise en œuvre dans le cas de sollicitations en fatigue, l'endommagement cumulé conduisant à la rupture ne pouvant être modélisé que par un code numérique en éléments-finis.
\end{abstract}

Mots clés : Essais accélérés / fatigue / méthode des éléments-finis / plan d'expériences / systèmes complexes

\begin{abstract}
Accelerated life testing for complex mechanical devices. This paper presents an accelerated test method applicable to complex mechanical devices when no analytical relationship with respect to the stress level can be defined. If a numerical approach remains possible, the numerical model can be fitted to the accelerated test results. Thus, long term failures can be predicted from very short tests. This method is carried out in the case of fatigue, the evolution of the damage leading to the failure having to be modeled by a numerical finite-elements method.
\end{abstract}

Key words: Accelerated life testing / fatigue / finite-elements model / design of experiments / complex systems

\section{Introduction}

L'évolution de la concurrence industrielle nécessite de pouvoir prévoir des niveaux de fiabilité de plus en plus rapidement. Ces prédictions sont souvent obtenues à partir d'essais de durées de vie accélérés, habituellement nommés ALT (pour Accelerated Life Testing). Ce type d'essai consiste à soumettre un échantillon à des niveaux de sollicitation $\left(S_{1}, S_{2}, \ldots\right)$ supérieurs au niveau nominal $S_{0}$ et à extrapoler au niveau nominal les distributions de durées de vie obtenues (Fig. 1).

La loi exprimant la durée de vie caractéristique (moyenne ou médiane) en fonction du niveau de sollicitation est appelée loi d'accélération.

Dans le cas de systèmes simples, les durées de vie à un niveau de sollicitation donné $S$ peuvent être distribuées

\footnotetext{
a Auteur pour correspondance :

pascal.lantieri@angers.ensam.fr
}

selon une loi de distribution connue et on peut par ailleurs exprimer l'évolution, en fonction du niveau $S$, d'une durée de vie caractéristique.

Pour des éprouvettes de fatigue sollicitées en dehors du domaine d'endurance par exemple, on peut admettre que les durées de vie sont distribuées selon une loi lognormale et que la durée de vie médiane $N$ évolue en fonction du niveau de sollicitation $S$ selon une loi puissance inverse, ce qui signifie que :

$$
N(S)=\frac{A}{S^{C}}
$$

où $A$ et $C$ sont des paramètres caractéristiques qui peuvent être estimés à partir d'essais à 2 niveaux sévérisés, $S$ désigne le niveau de déformation si la fatigue est oligocyclique et le niveau de contrainte s'il s'agit de fatigue limitée. 


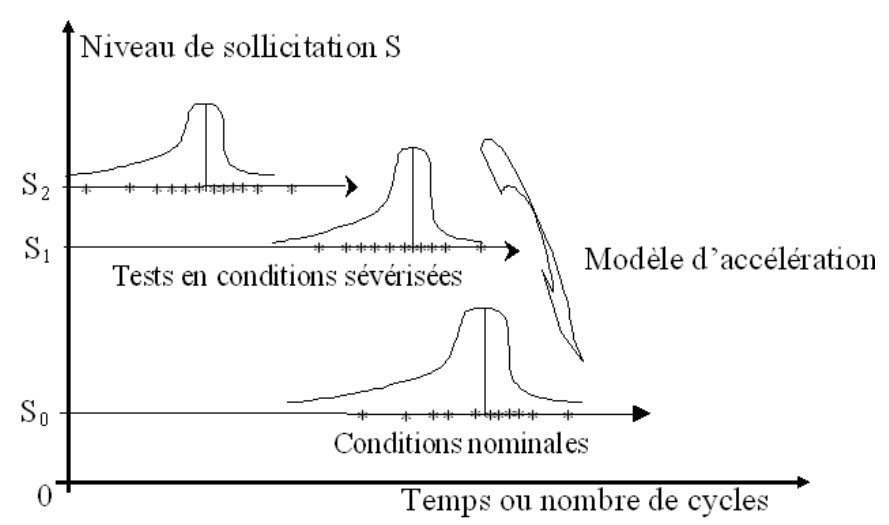

Fig. 1. Estimation de durées de vie par des essais accélérés (ALT).

De tels modèles ne peuvent plus être utilisés pour des géométries ou pour des modes de sollicitation plus complexes mais, faute de modèle analytique simple, on peut éventuellement recourir à des méthodes numériques.

La méthode proposée consiste à ajuster le modèle numérique utilisé aux durées de vie caractéristiques obtenues expérimentalement pour les niveaux sévérisés. Les techniques itératives usuelles de minimisation d'erreur (méthodes de descente) étant difficilement applicables pour des modèles purement numériques, nous suggérons plutôt de minimiser une approximation quadratique de cette erreur. Il suffit pour cela de recourir à un plan d'expériences numérique et de rechercher le minimum sur la surface de réponse obtenue.

\section{Méthode d'ajustement du modèle}

\subsection{Ajustement d'un modèle par méthode inverse}

Considérons une liste $\mathcal{M}$ de $N_{\text {mes }}$ mesures expérimentales sur un système et une liste $\mathcal{P}$ de $N_{\text {par }}$ paramètres d'un modèle de comportement de ce système.

Dans un problème direct, on connaît les valeurs des composantes de $\mathcal{P}$ et on cherche à prévoir la liste de mesures $\mathcal{M}$. On peut ainsi considérer que le problème direct est déterminé par un opérateur $F$ de l'ensemble des listes de paramètres $\mathcal{P}$ vers l'ensemble des mesures $\mathcal{M}$. Même si l'opérateur $F$ ne peut être explicité sous une forme simple, il pourra souvent être défini par une série de calculs.

Dans un problème inverse, on dispose de valeurs pour la liste de mesures expérimentales $\mathcal{M}$ et on cherche les valeurs de paramètres correspondantes. Si l'opérateur $F$ est bijectif et si les mesures sont déterminées avec précision, il suffit pour cela de définir l'opérateur inverse $F^{-1}$ (cf. Fig. 2).

Cela impose au minimum que $N_{\text {mes }}=N_{\text {par }}$ alors que, dans la pratique, on a souvent $N_{\text {mes }} \gg N_{\text {par }}$. Par ailleurs, toute mesure est nécessairement entachée d'erreurs. En général, on ne peut donc déterminer de solution exacte $F^{-1}(\mathcal{M})$. Il faut rechercher une solution approchée $\hat{\mathcal{P}}$

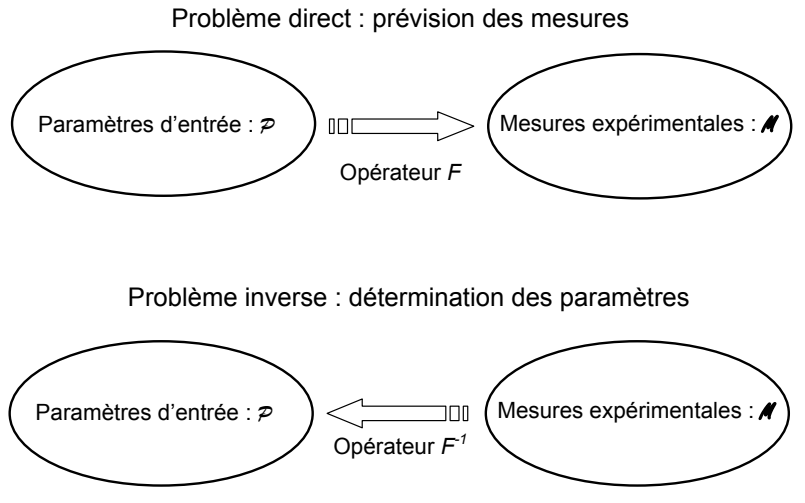

Fig. 2. Problèmes directs et inverses.

parmi les solutions du problème suivant :

$$
\left\{\begin{array}{l}
\phi(\hat{\mathcal{P}})=\operatorname{Min}_{\mathcal{P} \in R^{N_{\mathrm{par}}}} \phi(\mathcal{P}) \\
\phi(\mathcal{P})=\|F(\mathcal{P})-\mathcal{M}\|
\end{array}\right.
$$

\subsection{Minimisation de l'erreur $\phi$}

\subsubsection{Approximation quadratique de $\phi$}

Divers procédés existent pour résoudre le problème précédent, notamment les méthodes itératives dites de descente (gradient, Newton, etc.) consistant à s'approcher progressivement d'un minimum local de $\phi$ en partant d'un point supposé proche de ce minimum et en se déplaçant dans une direction appropriée. Cependant, ces méthodes ne permettent d'identifier que des minima locaux alors que le but est d'identifier le minimum global de $\phi$. Par ailleurs, elles nécessitent a priori de disposer d'expressions analytiques de $\phi$ pour pouvoir calculer des gradients, ce qui n'est pas le cas lorsqu'on ne dispose que d'un modèle numérique.

On peut y remédier en remplaçant $\phi$ par le polynôme de second degré de meilleure approximation quadratique d'une famille de $K$ points :

$$
\left\{\left(\mathcal{P}_{i}, \phi\left(\mathcal{P}_{i}\right)\right), i \in[1, K]\right\},
$$

$K$ étant supérieur au nombre de termes du polynôme. Le choix d'un polynôme de degré 2 garantit l'existence et l'unicité du minimum.

Pour que cette méthode d'ajustement soit précise, il convient de définir la liste des points $\mathcal{P}_{i}$ dans un domaine de valeurs réalistes encadrant au plus près la solution $\hat{\mathcal{P}}$ cherchée. Ce domaine peut être déterminé à partir de données bibliographiques et de calculs basés sur un modèle simplifié (calculs analytiques). Les estimations approximatives obtenues représentent le centre du domaine et les bornes sont estimées à partir des niveaux d'incertitude.

On peut vérifier que le domaine $\mathcal{D}$ encadre bien la solution en vérifiant que chacune des composantes du vecteur $F(\mathcal{P})-\mathcal{M}$ change de signe lorsque l'on parcourt le 
domaine $\mathcal{D}$. Une fois ce domaine défini et validé, il est judicieux de construire l'ensemble des points $\mathcal{P}_{i}$ selon un plan d'expériences adéquat.

\subsubsection{Construction et réalisation du plan d'expériences}

Le plan d'expériences adapté à la construction d'une surface de réponse quadratique est le plan composite centré de Box-Wilson [1]. Il est constitué d'un hypercube centré sur le domaine de valeurs explorées, complété par un point central et des points en étoile. Si, après normalisation des variables, chacune des faces de l'hypercube est à une distance $d$ de son centre, les points en étoile doivent être placés à une distance $\delta d$ garantissant l'isovariance par rotation des points du plan d'expériences. La distance $d$ doit être choisie de telle sorte que les points en étoile soient aux bornes du domaine.

Pour chacun des points $\mathcal{P}_{i}$ de ce plan, on effectue un calcul à l'aide du code numérique utilisé pour modéliser le système et on évalue pour chacun des niveaux sévérisés l'écart quadratique total $y$ entre les durées de vie estimées avec le modèle et les durées de vie caractéristiques expérimentales.

\subsubsection{Détermination du polynôme d'approximation de $\phi$ sur ce plan}

En notant $Y$ le vecteur des réponses $y_{i}$ obtenues pour chacun des points $\mathcal{P}_{i}$ et $X$ la matrice des valeurs prises par les diverses variables du polynôme en chacun de ces points, le problème revient à résoudre le système d'équations surdéterminé :

$$
Y=\Theta X
$$

où $\Theta$ est la liste de coefficients du polynôme cherché.

A priori, la meilleure solution de ce système est donnée par :

$$
\Theta=\left(X^{T} X\right)^{-1} X^{T} Y
$$

$T$ désignant l'opérateur transposé.

Cependant, afin d'éliminer les facteurs liés aux effets purement aléatoires induits par la démarche, il convient d'effectuer une analyse de variance (ANOVA) sur chacun des termes du polynôme obtenu $[1,2]$. En effet, même si en l'occurrence, le plan d'expériences est purement numérique, le fait d'approximer la réponse par un polynôme du second degré a des effets purement aléatoires. Comme il n'y a pas de raison de conserver les termes induits par ces effets, seuls les coefficients significativement non nuls (à un risque $\alpha$ usuellement choisi à la valeur $5 \%$ ) seront conservés.

Après chaque élimination de termes, une nouvelle analyse de variance doit être effectuée pour le polynôme tronqué retenu. Dès que l'analyse de variance assure que tous les coefficients conservés sont significativement non nuls, le polynôme $Q$ correspondant peut être considéré comme une approximation représentative de la réponse $y$. On peut donc considérer que les valeurs de paramètres minimisant ce polynôme sont des bonnes estimations des paramètres solutions du problème (2).

\subsubsection{Ajustement du modèle numérique et vérification}

On cherche la valeur de $\mathcal{P}$ pour laquelle le polynôme $Q$ est minimum, soit par une méthode de descente, soit par résolution de l'équation $\nabla Q=0$. Il convient alors de vérifier le bon ajustement aux données expérimentales du modèle numérique défini avec la solution $\hat{\mathcal{P}}$ obtenue. Pour cela, on peut évaluer l'hypothèse $\phi(\hat{\mathcal{P}})=0$ par un test unilatéral (à un risque $\alpha$ donné).

Si les durées de vie caractéristiques obtenues aux divers niveaux sévérisés sont des moyennes sur un nombre de mesures suffisamment grand, ces variables suivent des lois normales. La variable $\phi(\hat{\mathcal{P}})^{2}$ suit donc une loi du $\chi^{2}$ dont le nombre de degrés de liberté est égal au nombre de niveaux sévérisés. Il y aura par exemple 2 degrés de liberté s'il y a 2 niveaux $S_{1}$ et $S_{2}$ comme indiqué sur la figure 1.

\section{Application à un problème de fatigue}

\subsection{Construction du modèle}

Dans le cas d'une étude en fatigue, les mesures expérimentales les plus significatives et les plus simples à obtenir sont les nombres médians de cycles à rupture, également appelés durées de vie médianes en fatigue.

On peut estimer ces durées de vie à partir du dommage en chacun des points du système considéré. Lorsque la variation du niveau de contrainte reste identique à chaque cycle de sollicitation, le dommage, initialement nul, augmente d'un même incrément et on considère que l'on atteint la rupture dès qu'il prend la valeur 1 .

Ainsi, il suffit de connaître le dommage maximum $D_{\max }$ subi pour un seul cycle et la durée de vie médiane peut alors être estimée par :

$$
N=\frac{1}{D_{\max }}
$$

Ce dommage peut lui-même être estimé à partir des tenseurs de contraintes ou de déformations et de paramètres caractéristiques $a, b, c, \ldots$ du matériau. Les problèmes directs et inverses peuvent se modéliser selon la figure 2 avec :

$-\mathcal{P}=\{$ Paramètres caractéristiques du matériau $: a, b$, $c \ldots\}$;

- $\mathcal{M}=\left\{\right.$ Durées de vie en fatigue : $N_{1}, N_{2}, \ldots$ pour divers niveaux de déformations ou de contraintes $\}$.

L'opérateur $F$ doit, pour chacun des niveaux $S_{i}$ :

- évaluer les tenseurs de contrainte et de déformations ainsi que le dommage en tout point de la pièce et à tout instant du cycle;

- calculer le dommage maximum à la fin du cycle ;

- donner son inverse $N_{i}$.

Cet opérateur $F$ mettra donc en œuvre des calculs tensoriels en de nombreux points du système, ce qui doit en général être réalisé par un code numérique EF. 
Des modèles numériques adaptés à des calculs sur pièces ou systèmes mécaniques complexes existent, que ce soit pour des problèmes de fatigue dite oligocyclique où l'on provoque une déformation plastique du matériau à chaque cycle, ou pour des problèmes d'endurance où l'on ne dépasse jamais la limite élastique.

Cependant, dès que la partie sollicitée comporte la moindre singularité géométrique ou que le chargement du système est multiaxial, il devient difficile de définir $F$ par une expression analytique.

On peut tout de même utiliser un opérateur simplifié $F_{0}$ lorsque la seule partie sollicitée est assimilable à une poutre droite de section constante en sollicitation simple (traction compression, torsion, flexion, etc.). En effet, dans ce cas, il n'est pas nécessaire de résoudre des équations entre tenseurs définis en chaque point de la pièce mais uniquement des équations à une dimension de résistance des matériaux. On détermine ainsi directement la distribution des contraintes et la déformée (donc la valeur des déformations).

Dans des situations de ce type, on peut calculer $F_{0}^{-1}(\mathcal{M})$ par des méthodes analytiques simples et estimer ainsi les paramètres caractéristiques du matériau. Sachant que, dans la plupart des cas, les hypothèses requises pour ce type de calcul ne sont pas parfaitement justifiées, ces estimations doivent malgré tout être améliorées. Pour ce faire, nous proposons d'utiliser la méthode décrite dans la section précédente, cette méthode pouvant être développée selon l'algorithme suivant :

- calcul de $F_{0}^{-1}(\mathcal{M})$ et des niveaux de précision sur chaque coefficient;

- détermination du domaine $\mathcal{D}$ et des points $\mathcal{P}_{i}$ du plan composite centré dont les points en étoile sont aux limites du domaine $\mathcal{D}$;

- pour chacun des points du plan composite centré : mise en œuvre du code EF pour le calcul des dommages pour un cycle de sollicitation en tout point du système mécanique considéré, calcul du dommage maximum, estimation de la durée de vie caractéristique, calcul de l'erreur $\phi(\mathcal{P})$;

- détermination par la méthode des surfaces de réponse du polynôme de degré 2 de meilleure approximation de la réponse $y=\phi(\mathcal{P})$, élimination des termes non significatifs après ANOVA. Réitération de l'ANOVA jusqu'à obtention d'un polynôme $Q$ ne comportant que des termes significatifs;

- détermination de l'estimation $\hat{\mathcal{P}}$ par minimisation de $Q$;

- estimation de la liste des durées de vie $F(\hat{\mathcal{P}})$ avec le code EF et vérification de l'hypothèse $\phi(\hat{\mathcal{P}})=0$ par un test au risque $\alpha$.

Si le test d'hypothèse est positif, on peut considérer que le modèle est ajusté et le code EF correspondant peut être utilisé pour estimer la distribution de durées de vie au niveau de contrainte ou de déformation nominal.

Cette démarche peut être détaillée dans le cas d'essais accélérés sur attaches trombones.

\subsection{Expérience proposée}

Nous réalisons sur des échantillons de 50 trombones des séries d'ouvertures dans un sens puis dans l'autre jusqu'à la rupture. Le type de sollicitation $S$ considéré ici est la déformation angulaire imposée aux trombones au cours de torsions alternées successives. Les niveaux de sollicitation sévérisés choisis sont les valeurs suivantes de l'angle d'ouverture $\alpha$ du trombone :

$$
\alpha=90^{\circ} \text { pour } S_{1} \text { et } \alpha=180^{\circ} \text { pour } S_{2}
$$

À partir des durées de vie médianes (en nombres de cycles jusqu'à rupture) obtenues à ces deux niveaux, on cherche à estimer la distribution des durées de vie pour un angle d'ouverture du trombone $S_{0}=45^{\circ}$, ce niveau de sollicitation étant considéré comme le niveau nominal (Figs. 3 à 5).

Les nombres de cycles d'ouverture $n_{i}^{\exp }$ (entre la position fermée et le retour à cette position) obtenus jusqu'à la rupture pour les niveaux $S_{i}(i \in[0,2])$ sont indiqués dans le tableau A.1 porté en annexe A. Sachant qu'en général les ruptures se produisent en milieu de cycle, ces nombres sont a priori décimaux. Cependant, compte tenu des conditions expérimentales très imparfaites, une précision au-delà de l'unité ne serait pas significative.

Les niveaux de sollicitation imposés correspondent à de la fatigue oligocyclique, ce domaine permettant d'obtenir des durées de vie beaucoup plus courtes et donc de réaliser l'expérience plus rapidement que dans le domaine d'endurance.

Une discrétisation temporelle est nécessaire afin de tenir compte de l'apparition progressive d'un endurcissement au fur et à mesure que la déformation plastique augmente (modèle de Lemaître [3]). Ce modèle discret étant relativement complexe, il est utile d'obtenir au préalable une première estimation des paramètres à l'aide d'un modèle simplifié.

\subsection{Modèle de comportement simplifié}

\subsubsection{Hypothèses}

- La majeure partie de la déformation subie à chaque cycle est plastique.

- Seule la partie AB du trombone (repérée sur Fig. 4) se déforme.

- Cette partie AB se comporte comme une poutre droite encastrée en B (point de jonction avec la partie immobile du trombone).

\subsection{2 Équations simplifiées}

Sous ces hypothèses, il est possible d'utiliser :

- des méthodes de calcul en déformation de poutres;

- la loi de comportement en fatigue oligocyclique de Manson Coffin définie par [4] :

$$
N\left(\Delta \varepsilon^{\mathrm{pl}}\right)^{c}=\beta
$$




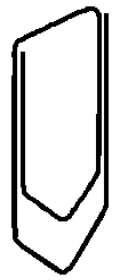

Fig. 3. Trombone (état initial).

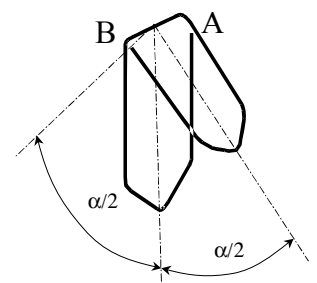

Fig. 4. Angle d'ouverture $\alpha$.

où $c$ et $\beta$ sont des constantes indéterminées, $\Delta \varepsilon^{\mathrm{pl}}$ est la valeur absolue maximum de la déformation plastique équivalente.

Comme la torsion atteint son maximum au point B, on peut en déduire que la première déformation plastique est située en $\mathrm{B}$, et donc que tout accroissement de la déformation se localise préférentiellement en ce point (zone la plus ductile). Ainsi, la totalité de la déformation angulaire imposée par l'opérateur a été attribuée à la déformation plastique au niveau de ce point $B$ et peut être donnée par [5]:

$$
\Delta \varepsilon^{\mathrm{pl}}=\sqrt{\frac{2}{3}} \gamma_{\mathrm{M}}
$$

où $\gamma_{\mathrm{M}}$ est la torsion maximale subie à chaque cycle de déformation (au bord de la section circulaire en B) définie par :

$$
\gamma_{\mathrm{M}}=R \frac{\alpha}{2 L}
$$

$R$ étant le rayon de la partie $\mathrm{AB}$ du trombone, $L$ étant la longueur de poutre, $\alpha$ étant l'angle d'ouverture illustré sur la figure 4 .

Des relations (6), (7) et (8), on déduit l'opérateur simplifié $F_{0}$ associant, à tout couple de paramètres $\{b, c\}$, la durée de vie médiane :

$$
N=\frac{b}{\alpha^{c}}
$$

où $b=\beta\left(\frac{L \sqrt{6}}{R}\right)^{c}$.

On obtient donc les premières estimations de paramètres :

$$
(\hat{b}, \hat{c})=F_{0}^{-1}\left(N_{1}, N_{2}\right)
$$

$N_{1}$ et $N_{2}$ étant les durées de vie médianes aux 2 niveaux de déformation sévérisés.

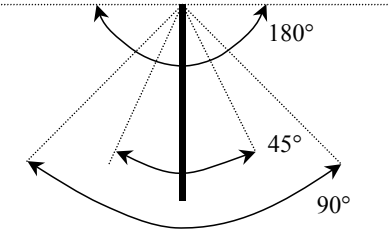

Fig. 5. Les 3 niveaux.

\subsection{Modèle amélioré}

\subsection{1 Équations locales}

Ce modèle est défini pour décrire l'accumulation d'endommagement conduisant finalement à la rupture. À partir de la notion de continuum de dommage $[3,6,7]$, la propagation d'une fissure peut être décrite en simulant la propagation d'une zone totalement endommagée.

Avec l'approche développée par Lemaitre [3], le problème consiste à résoudre les équations caractéristiques $\mathrm{du}$ comportement du matériau. Les équations du problème peuvent ainsi être obtenues à partir de :

- la décomposition de la déformation en une partie élastique et une partie plastique :

$$
\varepsilon=\varepsilon^{\mathrm{el}}+\varepsilon^{\mathrm{pl}}
$$

- la loi élastique couplée à l'endommagement (établie par Lemaitre) :

$$
\sigma_{i j}=(1-D)\left(\lambda \delta_{i j} \varepsilon_{k k}^{\mathrm{el}}+2 \mu \varepsilon_{i j}^{\mathrm{el}}\right)
$$

où $\lambda$ et $\mu$ sont les constantes de Lamé et où $\delta_{i j}$ est le symbole de Kronecker

- la loi d'endommagement :

$$
D=D\left(\sigma_{i j}, \varepsilon_{i j}, a_{k}\right)
$$

où le dommage dépend de coefficients intrinsèques $a_{k}$ du matériau

- la fonction de chargement de Von Mises couplée au dommage :

$$
f=\sigma_{\mathrm{eq}}-(1-D)\left(\sigma^{\mathrm{el}}+\sigma_{0}\right)
$$

où $\sigma_{\text {eq }}$ est la contrainte équivalente, $\sigma^{\mathrm{el}}$ désigne le tenseur de contraintes élastiques, $\sigma_{0}$ est la partie nonlinéaire donnée par la loi d'endurcissement :

$$
\sigma_{0}=K\left(\varepsilon_{\mathrm{eq}}\right)^{m}
$$

où $K$ et $m$ sont des paramètres caractéristiques du matériau, $\varepsilon_{\mathrm{eq}}=\sqrt{\varepsilon_{1}^{2}+\varepsilon_{2}^{2}+\varepsilon_{3}^{2}}$, les valeurs $\varepsilon_{i}$ représentant les valeurs propres du tenseur des déformations plastiques $\varepsilon^{\mathrm{pl}}$.

Le modèle plastique est complété par la description de l'évolution des variables caractéristiques du chargement avec l'accroissement progressif de la déformation plastique. 
Ces équations peuvent être notées :

$$
d H^{\alpha}=h^{\alpha}\left(d \varepsilon_{i j}, \sigma_{i j}, H^{\alpha}, D\right)
$$

où $H^{\alpha}(\alpha=1,2, \ldots, n)$ est une série de variables scalaires caractéristiques de l'état de chargement, $d H^{\alpha}$ et $d \varepsilon_{i j}$ désignent les différentielles des grandeurs $H^{\alpha}$ et $\varepsilon_{i j}, h^{\alpha}$ est la fonction d'endurcissement associée à $H^{\alpha}$.

\subsubsection{Intégration des équations locales}

Les méthodes d'intégration des équations nonlinéaires caractéristiques sont basées sur un algorithme spécifique permettant de résoudre les équations sous leur forme discrétisée [8-10].

À cet effet, on suppose que la totalité de l'incrément du champ de contrainte sur un petit intervalle de temps $\left[t_{n}, t_{n+1}\right]$ est purement élastique. Ainsi, on obtient la loi de comportement élastique suivante :

$$
\sigma_{n+1}^{T}=\sigma_{n}+\Delta \sigma=\left(1-D_{n}\right) C_{\mathrm{el}}\left[\left(\varepsilon_{\mathrm{tot}}\right)_{n+1}-\left(\varepsilon^{\mathrm{pl}}\right)_{n}\right]
$$

l'exposant $T$ signifiant qu'il ne s'agit que de valeurs testées et $C_{\mathrm{el}}$ étant l'opérateur élastique de Hooke.

Si le critère précédent de comportement élastique satisfait la condition de chargement $f<0$, la prédiction est exacte et la condition de chargement est donc validée. On peut alors établir que :

$$
\sigma_{n+1}=\sigma_{n+1}^{T}
$$

Dans le cas contraire, il faut ajouter une correction plastique basée sur un algorithme en boucle suivant lequel la correction est réajustée jusqu'à ce que les variables au temps $t_{n+1}$ satisfassent malgré tout la condition de chargement. Cela signifie que les lois d'évolution discrétisées et la loi élastique vérifient le système :

$$
\left\{\begin{array}{l}
f=0 \\
\sigma_{n+1}-\left(1-D_{n+1}\right) C_{\mathrm{el}}\left(\varepsilon_{n}+\Delta \varepsilon-\varepsilon_{n}^{\mathrm{pl}}-\Delta \varepsilon^{\mathrm{pl}}\right)=0 \\
\Delta H^{\alpha}=h^{\alpha}\left(\Delta \varepsilon_{i j},\left(\sigma_{i j}\right)_{n+1}, H_{i j}^{\alpha}, D_{n+1}\right)
\end{array}\right.
$$

La déformation plastique correspondante est obtenue à tout instant d'un cycle d'ouverture et de fermeture du trombone en résolvant ces équations en tout point du trombone à l'aide du code de calcul par EF.

\subsubsection{Calcul des différents dommages}

En négligeant la déformation élastique devant la déformation plastique, les dommages au cours d'un cycle peuvent être calculés pour tout niveau de déformation angulaire $S$ en considérant :

- le dommage dû à l'endurcissement [4] : $D_{1}=a S$;

- l'endommagement par fatigue oligo-cyclique : $D_{2}=$ $\frac{S^{c}}{b}$ où $a, b$ et $c$ sont des paramètres indéterminés.

On peut alors calculer l'endommagement total : $D=D_{1}+D_{2}$.

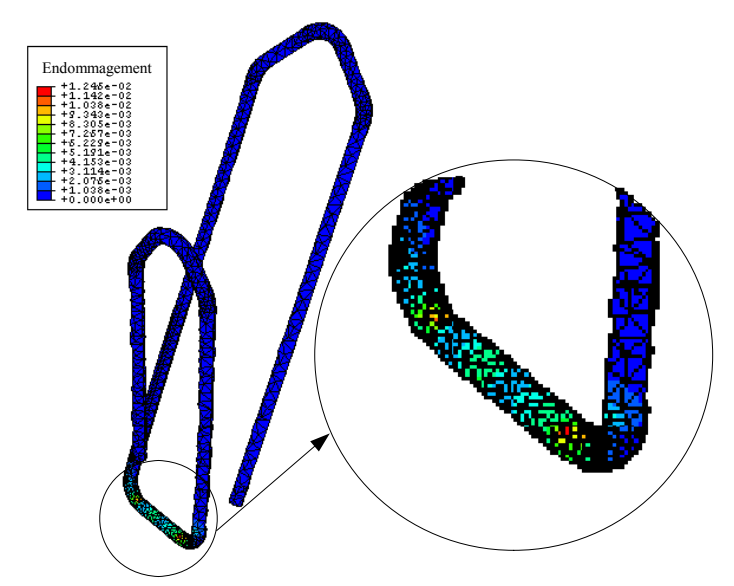

Fig. 6. Endommagement après 1 cycle d'ouverture.

\subsubsection{Modélisation en éléments-finis}

Les équations précédentes peuvent être résolues en décomposant en 5222 éléments 3D tétraédriques, le maillage étant fortement affiné dans les zones courbes.

Le modèle considère comme fixes tous les nœuds de la patte centrale du trombone (partie $\mathrm{AB}$ non comprise) et un déplacement en rotation d'axe $\mathrm{AB}$ de l'ensemble des nœuds de l'autre patte du trombone est imposé à chaque pas de temps.

- État initial : trombone ouvert (angle d'ouverture $-\alpha$, dommage initial négligé)

- Déplacements imposés à chaque pas de temps : $2 n_{\mathrm{t}}$ rotations d'angle $\alpha / n_{\mathrm{t}}$ (où $n_{\mathrm{t}}=120$ incréments de temps) pour passer à la position ouverte de l'autre côté, $2 n_{\mathrm{t}}$ rotations d'angle $-\alpha / n_{\mathrm{t}}$ pour le retour à la position initiale.

La figure 6 montre une déformée après un cycle d'ouverture avec indication des dommages aux divers nœuds du maillage, et donc du dommage $D_{\max }$.

La loi de Miner permet alors de déduire le nombre de cycles à rupture médian $N=1 / D_{\max }$.

On a ainsi défini l'opérateur $F$ associant à toute liste de paramètres matériau les durées de vie correspondantes aux deux niveaux sévérisés. Dans cet exemple, seuls les paramètres $a, b$ et $c$ ont été considérés indéterminés. Tous les autres ont été estimés par les valeurs figurant dans les bases de données sur l'acier utilisé.

\subsection{Résultats numériques}

Les diverses étapes résumées à la section 3.1 ont successivement conduit aux résultats suivants.

\subsubsection{Premières estimations $F_{0}^{-1}(\mathcal{M})$ et niveaux de précision}

À partir des valeurs expérimentales figurant sur le tableau A.1, on obtient la liste $\mathcal{M}$ des nombres de cycles médians :

- $N_{1}=7,2$ pour le niveau de déformation $S_{1}=90^{\circ}$;

- et $N_{2}=2,12$ pour le niveau $S_{2}=180^{\circ}$. 
Tableau 1. Niveaux des paramètres.

\begin{tabular}{|c|c|c|c|c|c|c|}
\hline \multirow[t]{2}{*}{ Paramètre } & \multirow{2}{*}{$\begin{array}{c}\text { Paramètre normalisé } \\
\text { associé }\end{array}$} & \multicolumn{5}{|c|}{ Niveaux du paramètre } \\
\hline & & $-\delta$ & -1 & 0 & 1 & $+\delta$ \\
\hline$a$ & $X_{1}$ & 0,1 & 0,26 & 0,5 & 0,74 & 0,9 \\
\hline$b$ & $X_{2}$ & 1,2 & 1,7 & 2,45 & 3,2 & 3,7 \\
\hline$c$ & $X_{3}$ & 0,3 & 0,4 & 0,55 & 0,7 & 0,8 \\
\hline
\end{tabular}

D'après les équations (9) et (10) avec $L=10 \mathrm{~mm}(=A B)$ et $R=0,5 \mathrm{~mm}$, on obtient : $\hat{b}=2,47( \pm 50 \%)$ et $\hat{c}=$ $0,56( \pm 50 \%)$.

Le paramètre $a$ a été estimé au centre de l'ensemble de ses valeurs possibles, soit l'intervalle $[0,1 ; 0,9]$. Ainsi : $\hat{a}=0,5( \pm 80 \%)$.

Comme il n'a pas été facile de connaître l'erreur induite par l'utilisation d'un modèle de poutre, les niveaux d'incertitude ont été choisis assez élevés.

\subsubsection{Domaine $\mathcal{D}$ et plan d'expériences}

Dans notre cas, $\mathcal{D}=[0,1 ; 0,9] \times[1,2 ; 3,7] \times[0,3 ; 0,8]$. Le point central du plan sera donc : $(0,5 ; 2,45 ; 0,55)$.

Les paramètres normalisés sont:

$$
X_{1}=\frac{a-0,5}{0,4}, X_{2}=\frac{b-2,45}{1,25} \text { et } X_{3}=\frac{c-0,55}{0,25}
$$

Dans cet espace de paramètres, le point central est $(0 ; 0$; 0 ) et les bornes du domaine doivent être $-\delta$ et $+\delta$ avec $\delta=1,682$ pour assurer l'isovariance par rotation, ce qui impose $d=1 / \delta=0,24$. Les niveaux de paramètres du plan sont donnés dans le tableau 1.

\subsubsection{Résultats du modèle EF pour ce plan d'expériences}

Pour chacun des 15 points $\mathcal{P}_{i}$ de ce plan, les dommages et nombres de cycles à ruptures peuvent être estimés à l'aide du code EF défini précédemment. Après vérification de l'encadrement de la solution cherchée (changement de signe de l'erreur absolue sur $\mathcal{D}$, on peut calculer les erreurs quadratiques totales $y_{i}=\phi\left(\mathcal{P}_{i}\right)$ en chacun des points du plan d'expériences (Tab. A.2).

\subsubsection{Polynôme de meilleure approximation $Q$}

Le polynôme cherché est de la forme :

$$
\begin{aligned}
Q\left(x_{1}, x_{2}, x_{3}\right)=a_{0}+\sum_{j=1}^{3} a_{j} x_{j}+\sum_{j=1}^{3} a_{j j} x_{j}^{2} & \\
& +\sum_{j=1}^{3} \sum_{i=1}^{j-1} a_{i j} x_{i} x_{j}
\end{aligned}
$$

En ajustant par moindres carrés aux valeurs de $y_{i}$ indiquées sur le tableau A.2, on obtient, avec un coefficient de détermination $R^{2}=0,94$, le polynôme $Q$ défini par :

$$
\begin{aligned}
& Q\left(x_{1}, x_{2}, x_{3}\right)=1,28+0,23 x_{1}-0,43 x_{2}-3,02 x_{3}+0,14 x_{1}^{2} \\
& \quad+0,087 x_{2}^{2}+2,79 x_{3}^{2}-0,018 x_{1} x_{2}-0,586 x_{1} x_{3}+0,077 x_{2} x_{3}
\end{aligned}
$$

Le test $F$ montre que la régression linéaire est significative au risque $5 \%$ mais les tests de Student sur les divers coefficients montrent que les 4 termes en $x_{1}, x_{1}^{2}, x_{1} x_{2}$ et $x_{2} x_{3}$ ne sont pas significatifs.

Sachant qu'en éliminant le terme en $x_{1}^{2}$, on ne garantit plus l'existence d'un minimum, on le conserve malgré tout dans un premier temps et on élimine uniquement les trois autres. Le polynôme de meilleure approximation dans la nouvelle base est, avec un coefficient de détermination peu dégradé $\left(R^{2}=0,92\right)$ :

$$
\begin{aligned}
T\left(x_{1}, x_{2}, x_{3}\right)=1,35 & -0,43 x_{2}-3,09 x_{3}+0,26 x_{1}^{2} \\
& +0,09 x_{2}^{2}+2,97 x_{3}^{2}-0,46 x_{1} x_{3}
\end{aligned}
$$

Le test $F$ montre que la régression obtenue est toujours significative au risque $5 \%$ et les tests de Student montrent que le risque de retenir à tort ces nouveaux termes est systématiquement inférieur à $5 \%$. Il reste cependant préférable de vérifier que la minimisation du polynôme $T$ conduit bien à un modèle EF représentatif des résultats d'essais.

\subsubsection{Estimation des paramètres}

Par gradient conjugué, on constate que $T$ prend une valeur minimum (voisine de 0 ) pour les valeurs de paramètres : $\hat{a}=0,49 ; \hat{b}=2,39$ et $\hat{c}=0,56$.

\subsubsection{Estimation des durées de vie par le modèle EF et vérification du bon ajustement}

Un calcul EF avec ces paramètres pour 1 cycle de déformation permet d'estimer le dommage maximum à : $55,8 \%$ pour le niveau $S_{1}$ et $10,2 \%$ pour le niveau $S_{2}$, ce qui correspond à des durées de vie médianes $N_{1}=1,79$ et $N_{2}=9,80$ alors que les valeurs expérimentales étaient 2,12 et 7,25 .

Sous l'hypothèse de bon ajustement, la variable $\sum \frac{\left(N_{i}^{\mathrm{EF}}-N_{i}^{\exp }\right)^{2}}{N_{i}^{\mathrm{EF}}}$ suit une loi du $\chi^{2}$ à 2 degrés de liberté et reste donc en dessous du seuil de 5,99 dans $95 \%$ des cas. Or, elle prend la valeur 0,72 .

Ainsi, les écarts entre les résultats du modèle EF et les résultats expérimentaux ne sont pas significatifs et il n'y a donc pas lieu de rejeter l'hypothèse de bon ajustement du modèle EF. Dans le cas présent, il est donc inutile de modifier à nouveau les paramètres du polynôme $T$.

Ce modèle ajusté permet d'estimer la durée de vie médiane pour $\alpha=45^{\circ}$ à :

$$
\hat{N}_{0}=1 / D=11,4 \text { cycles }
$$

(la valeur expérimentale étant de 12 cycles). 


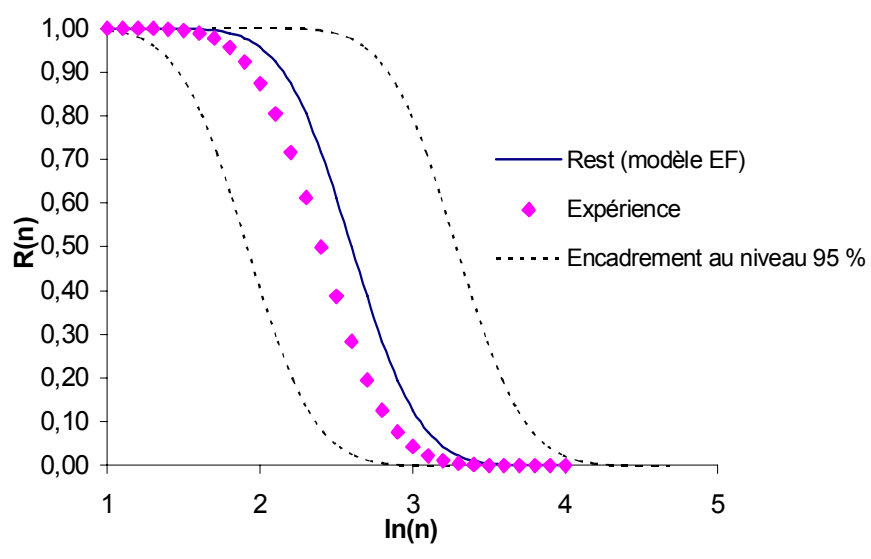

Fig. 7. Fonction de survie expérimentale et fonction estimée par EF.

\section{Validation de la méthode}

Dans le domaine de fatigue considéré, le nombre de cycles à rupture $n$ suit une loi log-normale de paramètres $\mu$ et $s$. L'écart-type $s$ de $\ln (n)$ peut être supposé identique pour tout niveau de déformation [11].

Au niveau de déformation nominal $\alpha=45^{\circ}$, il peut donc être estimé à partir des résultats aux 2 niveaux sévérisés.

D'autre part, la moyenne $\mu$ de $\ln (n)$ est déduite de la médiane $N_{0}$ de $n$ par la relation :

$$
\mu=\ln \left(N_{0}\right)-0,5 s^{2}
$$

On en déduit les estimations : $\mu=2,37$ (alors que la valeur expérimentale est de 2,4 ) et $\hat{s}=0,35$.

Pour le niveau nominal $\alpha=45^{\circ}$, la fonction de survie expérimentale $R_{\text {exp }}$, la fonction de survie $R_{\text {est }}$ estimée par EF ainsi que ses bornes au niveau de confiance $95 \%$ sont représentées figure 7 .

On constate que la démarche mise en ouvre aurait conduit à un encadrement satisfaisant de la fonction de survie obtenue en réalisant l'expérience au niveau nominal.

\section{Conclusion et perspectives}

En ajustant des paramètres caractéristiques d'un système mécanique jusqu'à ce que des durées de vie estimées par calcul numérique soient proches des durées de vie obtenues par essais accélérés, il est possible de prévoir en très peu de temps la fiabilité sur le long terme. Cette méthode permet d'effectuer les essais accélérés sur un seul modèle de prototype et de pouvoir modifier à loisir un système nouvellement conçu en estimant à chaque fois et instantanément le niveau de fiabilité.

Pour des pièces simples, le temps de calcul nécessaire pour cet ajustement peut être réduit en recourant à une première estimation basée sur un modèle simplifié. Cela n'est pas toujours possible mais la méthode présentée reste de toute façon applicable.

À condition de disposer de capacités de calcul suffisantes, on peut toujours construire un plan d'expériences numériques et ajuster les erreurs d'estimation des durées de vie par un polynôme de degré 2 , ce qui permet dans tous les cas de minimiser l'erreur liée à la modélisation numérique. Ainsi, quelle que soit la complexité du modèle, tant qu'une modélisation numérique est envisageable, la méthode d'essai de fatigue accélérée proposée est applicable.

La démarche a pu être illustrée dans le cas de tests en fatigue oligocyclique mais il serait intéressant de l'appliquer également à l'estimation de durées de vie dans d'autres domaines de fatigue. Pour ces diverses situations, on pourra améliorer les procédés d'optimisation évoqués.

\section{Références}

[1] G. Box et al., On the experimental attainment of optimum conditions, J. Royal Stat. Soc. 1 (1951) 1-45

[2] J. Cornell, How to Apply Response Surface Methodology, ASQC, 1990

[3] J. Lemaitre, A continuous damage mechanics model for ductile fracture, J. Eng. Materials and Technology 107 (1985) 83-89

[4] C. Bathias, J.-P. Baïlton, La fatigue des matériaux et des structures, Édition Hermes, 1997

[5] D. François, Élasticité et plasticité, Hermes, 1992

[6] J. Chaboche, Continuous damage mechanics - A tool to describe phenomena before crack initiation, Nucl. Eng. Design 64 (1981) 233-247

[7] A.L. Gurson, Continium theory of ductile rupture by void nucleation and growth, J. Eng. Materials and Technology 99 (1977) 2-15

[8] O. Sidebottom, C. Gebbhardt, Elastic springback in plates and beams formed by bending, Experiment Mechanics 19 (1979) 635-650

[9] Wilson et al., Die design handbook, American Society of tool and manufacturing engineers, 1965

[10] ABAQUS - HKS theory manual - Version 6.4

[11] O. Tebbi, F. Guerin, B. Dumon, Standard accelerated life testing model applied to mechanical components, J. IEST 48 (2005) 103-114 


\section{Annexe A}

Tableau A.1. Durées de vie expérimentales pour $\alpha=90^{\circ}, 180^{\circ}$ (ALT) et pour $\alpha=45^{\circ}$ (niveau nominal).

\begin{tabular}{cccccc}
\hline$N-45^{\circ}$ & $N-90^{\circ}$ & $N-180^{\circ}$ & $N-45^{\circ}$ & $N-90^{\circ}$ & $N-180^{\circ}$ \\
\hline 5 & 3 & 2 & 12 & 7 & 5 \\
5 & 4 & 2 & 12 & 7 & 5 \\
6 & 4 & 2 & 12 & 7 & 5 \\
6 & 4 & 2 & 13 & 7 & 5 \\
6 & 4 & 2 & 13 & 7 & 6 \\
7 & 5 & 4 & 13 & 8 & 6 \\
7 & 5 & 4 & 14 & 8 & 6 \\
8 & 5 & 4 & 14 & 8 & 6 \\
8 & 5 & 4 & 14 & 8 & 6 \\
8 & 5 & 4 & 14 & 8 & 6 \\
8 & 5 & 4 & 15 & 8 & 6 \\
8 & 6 & 4 & 15 & 8 & 6 \\
9 & 6 & 4 & 15 & 8 & 6 \\
9 & 6 & 4 & 15 & 8 & 6 \\
9 & 6 & 4 & 15 & 9 & 6 \\
9 & 6 & 4 & 16 & 9 & 6 \\
9 & 6 & 4 & 16 & 9 & 6 \\
9 & 6 & 4 & 17 & 10 & 7 \\
9 & 6 & 5 & 18 & 11 & 7 \\
10 & 6 & 5 & 18 & 11 & 7 \\
10 & 6 & 5 & 18 & 12 & 7 \\
10 & 6 & 5 & 18 & 11 & 7 \\
11 & 6 & 5 & 21 & 13 & 8 \\
11 & 7 & 5 & 23 & 13 & 8 \\
11 & 7 & 5 & & & \\
11 & 7 & 5 & & & \\
\hline & & 5 & & & \\
\hline
\end{tabular}

Tableau A.2. Durées de vie médianes estimées par la méthode EF et calculs des réponses $y_{i}$.

\begin{tabular}{|c|c|c|c|c|c|c|c|c|c|c|}
\hline \multirow[t]{2}{*}{$i$} & \multirow[t]{2}{*}{$a$} & \multirow[t]{2}{*}{$b$} & \multirow[t]{2}{*}{$c$} & \multicolumn{2}{|c|}{ Niveau $S_{1}$} & \multicolumn{2}{|c|}{ Niveau $S_{2}$} & \multirow{2}{*}{$\begin{array}{c}\text { Écarts } \\
\text { quadratiques }\end{array}$} & \multirow{2}{*}{$\begin{array}{l}\text { Approximations } \\
\qquad(a, b, c)\end{array}$} & \multirow{2}{*}{$\begin{array}{r}\text { Erreur }^{2} \\
\times 10^{-3}\end{array}$} \\
\hline & & & & $D(\%)$ & $N^{\mathrm{EF}}$ & $D(\%)$ & $N^{\mathrm{EF}}$ & & & \\
\hline 1 & 0,74 & 3,20 & 0,70 & 13,42 & 7,45 & 41,9 & 2,38 & 0,11 & 0,12 & 0,02 \\
\hline 2 & 0,26 & 3,20 & 0,70 & 13,34 & 7,50 & 41,0 & 2,44 & 0,17 & 0,16 & 0,01 \\
\hline 3 & 0,74 & 3,20 & 0,40 & 14,37 & 6,96 & 54,9 & 1,82 & 0,17 & 0,16 & 0,25 \\
\hline 4 & 0,26 & 3,20 & 0,40 & 14,21 & 7,04 & 54,8 & 1,82 & 0,13 & 0,12 & 0,14 \\
\hline 5 & 0,74 & 1,70 & 0,70 & 13,58 & 7,36 & 42,4 & 2,36 & 0,07 & 0,06 & 0,04 \\
\hline 6 & 0,26 & 1,70 & 0,70 & 13,42 & 7,45 & 42,3 & 2,36 & 0,10 & 0,10 & 0,00 \\
\hline 7 & 0,74 & 1,70 & 0,40 & 14,40 & 6,95 & 53,5 & 1,87 & 0,16 & 0,14 & 0,24 \\
\hline 8 & 0,26 & 1,70 & 0,40 & 14,24 & 7,02 & 53,4 & 1,87 & 0,11 & 0,09 & 0,48 \\
\hline 9 & 0,50 & 3,70 & 0,55 & 14,50 & 6,90 & 51,8 & 1,93 & 0,16 & 0,17 & 0,05 \\
\hline 10 & 0,50 & 1,20 & 0,55 & 13,50 & 7,41 & 42,4 & 2,36 & 0,08 & 0,10 & 0,35 \\
\hline 11 & 0,50 & 2,45 & 0,30 & 14,35 & 6,97 & 54,4 & 1,84 & 0,16 & 0,19 & 0,91 \\
\hline 12 & 0,50 & 2,45 & 0,80 & 13,35 & 7,49 & 40,9 & 2,45 & 0,16 & 0,16 & 0,02 \\
\hline 13 & 0,90 & 2,45 & 0,55 & 13,97 & 7,16 & 45,9 & 2,18 & 0,01 & 0,02 & 0,12 \\
\hline 14 & 0,10 & 2,45 & 0,55 & 13,71 & 7,29 & 45,8 & 2,19 & 0,01 & 0,02 & 0,20 \\
\hline \multirow[t]{2}{*}{15} & 0,50 & 2,45 & 0,55 & 13,83 & 7,23 & 45,8 & 2,18 & 0,00 & 0,00 & 0,02 \\
\hline & & & & & \multicolumn{3}{|c|}{$\begin{array}{c}\text { Écart quadratique } \\
\text { total }\end{array}$} & 1,6079 & 1,6079 & 2,86 \\
\hline
\end{tabular}

